\title{
Glucocorticoid Toxicity in the Hippocampus: Reversal by Supplementation with Brain Fuels
}

\author{
Robert M. Sapolsky \\ Clayton Foundation Laboratories for Peptide Biology, The Salk Institute for Biological Studies, San Diego, \\ California 92138
}

\begin{abstract}
Glucocorticoids (GCs) can damage neurons of the hippocampus, the principal target tissue in the brain for the hormone. Hippocampal neuron loss during aging in the rat is accelerated by prolonged GC exposure and decelerated by adrenalectomy. GCs appear to damage these neurons indirectly by inducing a state of vulnerability and thus impairing their capacity to survive a variety of metabolic challenges. As such, high physiological concentrations of the steroid increase hippocampal damage induced by an antimetabolite toxin, an excitotoxin, or hypoxiaischemia. Conversely, adrenalectomy attenuates the damage caused by these insults.

This study suggests that GCs endanger hippocampal neurons by impairing their energy metabolism. Neurons are extremely vulnerable to such disruption, all the insults potentiated by GCs either impair energy production or pathologically increase energy consumption, and GCs inhibit glucose utilization in the hippocampus. Administration of different brain fuels-glucose, mannose, fructose, or the ketone beta-hydroxybutyrate-reduced hippocampal damage induced by coadministration of GCs and either of 2 different neurotoxins (kainic acid and 3-acetylpyridine). This appeared to be due to a reduction in the damaging synergy between GCs and the toxin; as evidence, a dose of mannose that attenuated damage induced by kainic acid plus GCs failed to reduce damage induced by the same dose of kainic acid alone. Glucose (whose utilization is noncompetitively inhibited by GCs) and fructose (which does not readily penetrate the blood-brain barrier) were less effective at reducing damage than the other 2 fuels.
\end{abstract}

Glucocorticoids (GCs) are secreted by the adrenal cortex and mediate adaptation to acute stress (Munck et al., 1984). However, chronic GC exposure, whether a result of prolonged stress or pathological GC hypersecretion, can be profoundly deleterious, due in large part to the catabolic nature of the hormone's actions (Krieger, 1982; Munck et al., 1984). Among their injurious effects, GCs can damage neurons of the hippocampus. This has been demonstrated using pharmacologic GC concentrations (Aus der Muhlen and Ockenfels, 1969). GCs also have been implicated in the normative loss of hippocampal neurons during senescence, as basal titers of GCs rise with age in the rat (De Kosky et al., 1984; Landfield et al., 1978; Sapolsky et al., 1983a; Tang and Phillips, 1978) and mid-age adrenalectomy prevents such neuron loss (Landfield et al., 1981). Furthermore, sustained exposure to elevated concentrations of GCs acceler-

Received Aug. 12, 1985; revised Jan. 15, 1986; accepted Jan. 15, 1986.

The author gratefully acknowledges the technical assistance of Taide Pereyra, the manuscript assistance of Pamela Holand, and the advice of Lewis Krey, Abel Lajtha, Bruce McEwen, Paul Plotsky and Wylie Vale. The author is a Mathers Fellow of the Life Sciences Research Foundation.

Correspondence should be addressed to Robert M. Sapolsky at the above address.

Copyright (c) 1986 Society for Neuroscience $0270-6474 / 86 / 082240-05 \$ 02.00 / 0$ ates such hippocampal aging (Sapolsky et al., 1985). The anatomical specificity of such damage most likely arises from the high concentrations of GC receptors found throughout the hippocampus, which are unmatched in any other brain region (McEwen et al., 1968).

No studies have demonstrated that physiological concentrations of GCs can be directly toxic to hippocampal neurons. However, recent work suggests that GCs can induce a state of vulnerability in these neurons, which are then less likely to survive a broad range of metabolic insults. This "modulatory" hypothesis is based on the following observations: A variety of insults that damage the hippocampus have their potencies attenuated in adrenalectomized rats and exacerbated in rats with GC concentrations elevated to the upper physiological range (Sapolsky, 1985a, b; Sapolsky and Pulsinelli, 1985; Thoeret et al., 1985; Sapolsky, in press). These insults include hypoxiaischemia following vascular occlusion, and microinfusion of the excitotoxin kainic acid (KA) or of the antimetabolite 3-acetylpyridine (3AP). The insults differ in their mechanisms of action and regions of the hippocampus preferentially damaged. The steroids appear to act by impairing the capacity of neurons to survive the insults, rather than by increasing the intrinsic toxicity of the insults themselves. As evidence, the synergy between GCs and these insults occurs exclusively (in the casc of KA) or most dramatically (with $3 \mathrm{AP}$ and hypoxiaischemia) in cells with high concentrations of $\mathrm{GC}$ receptors. These observations suggest that GCs induce a broadly catabolic state in hippocampal neurons, diminishing their viability. Furthermore, that adrenalectomy reduced hippocampal damage below "normative" levels seen after these insults suggests that even basal concentrations of GCs could be deleterious to the hippocampus via this modulatory mechanism.

The present study demonstrates an intervention that diminishes the synergy between GCs and these insults. A hallmark of GC action is its inhibition of glucose uptake by peripheral target tissues (Munck, 1971). Such effects extend to the brain, particularly the hippocampus (Landgraf et al., 1978; M. Kadekaro, personal communication). Furthermore, neurons are markedly dependent on glucose as an energy substrate, given their extremely limited capacity for glycogen storage, as well as the limited number of energy sources that can penetrate the bloodbrain barrier (Siesjo, 1978). Finally, the 3 hippocampal insults whose potencies are modulated by GC milieu either impair the capacity of hippocampal neurons to generate energy or place undue demands on these neurons for energy: 3AP disrupts the electron transport chain and inhibits ATP synthesis (Hicks, 1955); ischemia appears to deplete neurons of ATP (Siesjo, 1981); and KA hyperexcites hippocampal neurons, most likely through its interaction with the glutamate neurotransmitter system (Coyle, 1983). These observations suggested that GCs, through their catabolic effects on neuronal energy metabolism, exacerbate the state of energy depletion in hippocampal neurons induced by these different insults, and thus increase their toxicity. The pres- 


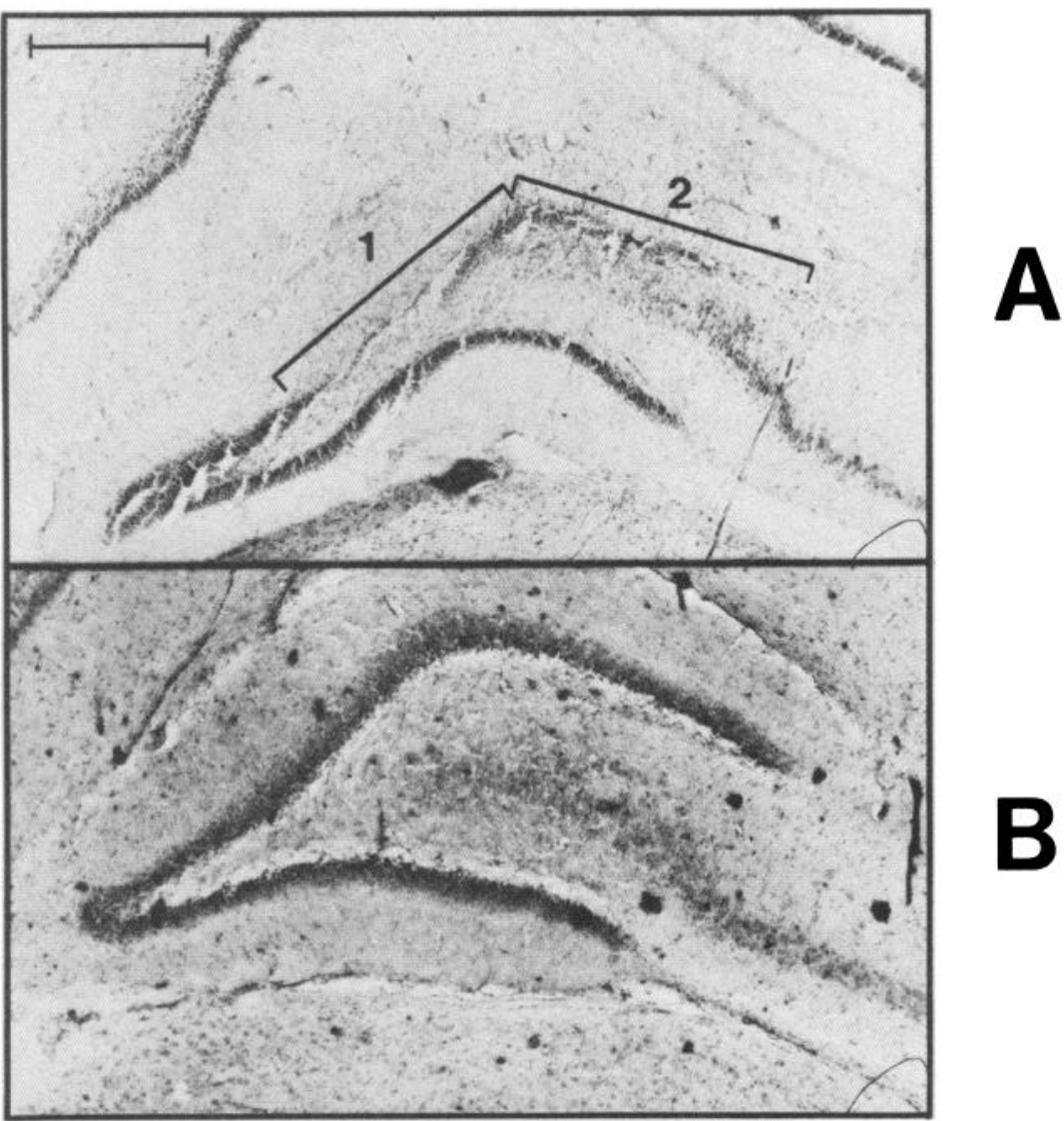

Figure 1. Representative photomicrographs showing typical hippocampal damage. $A, 3$ AP plus CORT administration. Note the extensive damage to the dorsal blade of the dentate gyrus. Damage to the first span of cells was $1.3 \times 0.15 \mathrm{~mm}$ long; damage to the second span of cells was $1.2 \times 0.16 \mathrm{~mm}$. Scale, $0.75 \mathrm{~mm}$. $B$, 3 AP plus CORT plus B-OH. No damage was noted in the dentate gyrus.

ent report demonstrates that increasing the availability of brain fuels to the hippocampus attenuates the synergy between GCs and these insults.

\section{Materials and Methods}

Male 3-month-old Sprague-Dawley rats (Charles River) were housed on a 12:12 light: dark cycle and given access to food and water ad libitum. In the first experiment, the hippocampus was damaged with the neurotoxin 3AP (Sigma, St. Louis). Toxin, $0.15 \mu \mathrm{l}$, in $0.85 \mu \mathrm{l}$ acidified saline ( $300 \mu \mathrm{g}$ ascorbic acid/ml saline) was infused unilaterally in Ammon's horn of the hippocampus at the coordinates AP 4.1, ML 2.1, and DV 3.0 (height of lambda set equal to bregma). This volume of toxin was just below threshold for producing consistent damage. In one group, rats were administered corticosterone (CORT; Sigma), the species-typical GC of rats, for $24 \mathrm{hr}$ before and after 3AP infusion. Rats were injected subcutaneously (s.c.) with $10 \mathrm{mg}$ CORT in $1 \mathrm{ml}$ sesame oil, a treatment that, for the greater part of a day, produces circulating CORT concentrations equivalent to those seen after major stressors (Sapolsky et al., 1985). Such elevated CORT concentrations produced elevated circulating glucose concentrations (Table 1), as determined by the glucose oxidase method with a Beckman glucose analyzer. In other rats, various sugars were coadministered with CORT in an attempt to reverse the synergy between CORT and 3AP. Rats were injected intraperitonally (i.p.) with either 0.5 or $1.0 \mathrm{gm}$ glucose, mannose, or fructose (Sigma) in $5 \mathrm{ml}$ saline every $3 \mathrm{hr}$ for $24 \mathrm{hr}$ before and after $3 \mathrm{AP}$ infusion. Administration of glucose (but not mannose or fructose) produced sustained elevations of circulating glucose concentrations above those seen in rats administered CORT alone (Table 1). Control rats received i.p. injections of saline.

Seven days after $3 \mathrm{AP}$ infusion, rats were perfused with $10 \%$ buffered formalin. Brains were removed and sectioned; $40 \mu \mathrm{m}$ coronal slices were retained every $320 \mu \mathrm{m}$, with the exception of the area of injection, where alternate sections were retained for confirmation of correct placement of the injection site. Only animals with tracks terminating in Ammon's horn were included in analysis. Sections were stained with cresyl violet and examined at $40 \times$ magnification. Hippocampal cell fields were identified by the anatomical criteria of Lorente de No (1934). The area of damage in each coronal section was determined by measuring the length and width of damage with a calibrated ocular grid. Areas of damage in successive coronal sections were then used to determine the total volume of damage with an AREA/vOLUME program on an IBM PC.

In the second experiment, rats were infused with $3 \mathrm{AP}$ and, in some cases, injected daily with CORT for $3 \mathrm{~d}$ beginning at the time of $3 \mathrm{AP}$ infusion. An attempt was made to reverse the CORT/3AP synergy with intracerebroventricular administration of the ketone beta-hydroxybutyrate (B-OH; Sigma). A 27 gauge cannula was stereotaxically placed in the lateral ventricle contralateral to the side of the toxin infusion at the coordinates AP 6.8, ML 1.2, DV 3.0. The cannula was cemented in place and attached by PE- 60 tubing to an Alzet minipump implanted

Table 1. Effects of corticosterone, glucose, mannose, and fructose administration on circulating glucose concentrations

Circulating glucose concentration $(\mathrm{mg} / \mathrm{ml}$ above baseline of $75 \pm 4)$

\begin{tabular}{lclcc}
$\begin{array}{l}\text { Time } \\
(\mathrm{hr})\end{array}$ & CORT & $\begin{array}{l}\text { CORT }+ \\
\text { glucose }\end{array}$ & $\begin{array}{l}\text { CORT }+ \\
\text { mannose }\end{array}$ & $\begin{array}{l}\text { CORT }+ \\
\text { fructose }\end{array}$ \\
\hline 1.5 & $1 \pm 8$ & $26 \pm 7$ & $1 \pm 2$ & $3 \pm 2$ \\
3 & $37 \pm 8^{*}$ & $60 \pm 3^{* *}$ & $15 \pm 6$ & $28 \pm 2^{*}$ \\
4.5 & $26 \pm 3^{*}$ & $65 \pm 13^{* *} \dagger$ & $14 \pm 6$ & $31 \pm 5^{*}$ \\
6 & $21 \pm 5$ & $72 \pm 7^{* *} \dagger$ & $21 \pm 3$ & $24 \pm 6$ \\
12 & $28 \pm 3^{*}$ & $70 \pm 5^{* *} \dagger$ & $19 \pm 4$ & $26 \pm 3$ \\
18 & $21 \pm 6$ & $68 \pm 9^{* *} \dagger$ & $23 \pm 3$ & $20 \pm 4$
\end{tabular}

Rats were injected s.c. with $10 \mathrm{mg}$ CORT in $1 \mathrm{ml}$ sesame oil and injected i.p. with $0.5 \mathrm{gm}$ of the sugar in $5 \mathrm{ml}$ saline; blood was taken from tail veins and analyzed for glucose with a Beckman glucose analyzer. $n=3-6$ for each group; separate rats were used for each time point. Asterisks, significant difference from basal values at the $0.05\left(^{*}\right)$ and $0.001\left(^{* *}\right)$ level; dagger, significant difference also from CORT-treated values at the 0.001 level (Scheffe post hoc test following 1-way ANOVA). 


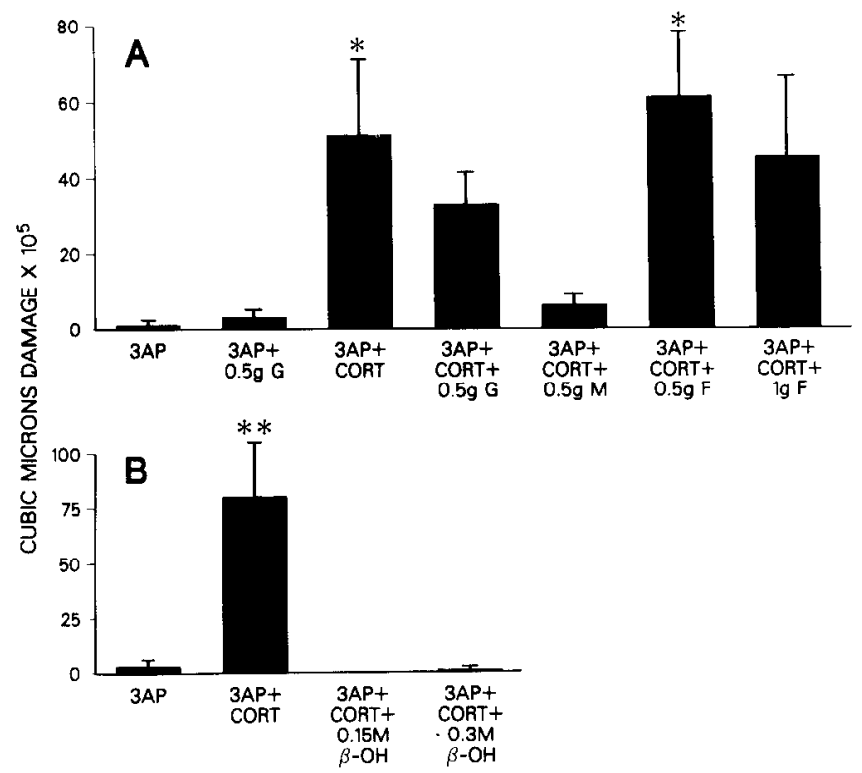

Figure 2. Potentiation of 3AP-induced hippocampal damage by CORT administration and reversal of this potentiation with brain fuels. Volumes of damage from entire unilateral hippocampi are presented. $A$, CORT, $10 \mathrm{mg}$, injected $24 \mathrm{hr}$ before and at the time of 3AP infusion ( $G$, glucose; $M$, mannose; $F$, fructose). Dosages indicate quantities of sugars injected i.p. every $3 \mathrm{hr}$ for $24 \mathrm{hr}$ before and after $3 \mathrm{AP}$ infusion. Sample sizes: $3 \mathrm{AP}$ (15 rats); $3 \mathrm{AP}+0.5 \mathrm{gm} \mathrm{G}(5)$; $3 \mathrm{AP}+$ CORT (15); $3 \mathrm{AP}+$ CORT + 0.5 gm G (10); 3AP + CORT + $0.5 \mathrm{gm} \mathrm{M}(9) ; 3 \mathrm{AP}+$ CORT + $0.5 \mathrm{gm} \mathrm{F}(6) ; 3 \mathrm{AP}+\mathrm{CORT}+1 \mathrm{gm} \mathrm{F}(8) . B$, CORT, $10 \mathrm{mg}$, injected every $24 \mathrm{hr}$ for $3 \mathrm{~d}$, beginning at the time of $3 \mathrm{AP}$ infusion. Dosage indicates the concentrations of $\mathrm{B}-\mathrm{OH}$ solutions with which the osmotic minipumps were filled. Sample sizes: 3AP (6 rats); 3AP + CORT (5); 3AP + CORT + $0.15 \mathrm{M} \mathrm{B-OH} \mathrm{(4);} 3 \mathrm{AP}+\mathrm{CORT}+0.3 \mathrm{M}$ $\mathrm{B}-\mathrm{OH}(5)$. Asterisks indicate significant differences from controls (administered 3AP alone) at $0.05\left(^{*}\right)$ and $0.01\left(^{* *}\right)$ levels, using Scheffe post hoc test following 1-way ANOVA.

s.c. between the shoulder blades. Pumps were filled with 0.15 or 0.30 $\mathrm{M}$ B-OH dissolved in saline, which delivered 1.5 or $3.0 \times 10^{-7} \mathrm{~mol}$ $\mathrm{B}-\mathrm{OH} / \mathrm{hr}$, rates previously shown to be effective in altering cerebral metabolism and ingestive behavior (Davis et al., 1981). Rats were cannulated immediately after $3 \mathrm{AP}$ infusion; control rats were fitted with cannulas and infused with saline. After $3 \mathrm{~d}$, the PE- 60 tubing was severed and closed. Subjects were killed 1 week after surgery, and 3AP-induced damage was quantified as described above. Photomicrographs are presented (Fig. 1) demonstrating 3AP-induced damage and the method of quantifying it.

In the third experiment, rats were infused in the hippocampus with $0.035 \mu \mathrm{g}$ of the neurotoxin KA (Sigma), with preparation of the toxin and stereotaxic surgery as described for 3AP. This dose reliably produces small amounts of damage, predominantly in the CA3 region of the hippocampus. Rats were adrenalectomized a week before surgery. Some rats were injected with CORT $24 \mathrm{hr}$ before and after surgery, and others were injected with both CORT and one of the sugars, as described. Rats were killed a week after surgery and processed as with 3AP rats.

Data were analyzed by 1 - and 2-way ANOVA, followed by Scheffe post hoc tests.

\section{Results}

An infusion of $0.15 \mu \mathrm{l}$ of $3 \mathrm{AP}$ was just below threshold for reliably producing hippocampal damage (Fig. $2, A, B$ ). Elevation of CORT concentrations during the $24 \mathrm{hr}$ bracketing the $3 \mathrm{AP}$ infusion significantly potentiated the volume of hippocampal damage induced by 3 AP. Such damage was centered in the dorsal blade of the dentate gyrus and was attributable to enhanced anterior-posterior and medial-lateral spread of damage.

The potentiation of $3 \mathrm{AP}$ damage by CORT was prevented by supplementation of rats with high concentrations of a number
Table 2. KA-induced hippocampal damage and modulation by CORT administration and supplementation with brain fuels

\begin{tabular}{lcl} 
& \multicolumn{2}{c}{ Damage $\left(\mu \mathrm{m}^{3} \times 10^{5}\right)$} \\
\cline { 2 - 3 } Group & $\begin{array}{l}\text { CA3 cell } \\
\text { field }\end{array}$ & $\begin{array}{l}\text { Total } \\
\text { hippocampus }\end{array}$ \\
\hline KA $(n=8)$ & $75 \pm 18$ & $166 \pm 41$ \\
KA + glucose $(n=8)$ & $106 \pm 21$ & $145 \pm 43$ \\
KA + mannose $(n=5)$ & $91 \pm 15$ & $126 \pm 42$ \\
KA + CORT $(n=8)$ & $176 \pm 12^{*}$ & $288 \pm 32^{*}$ \\
KA + CORT + glucose $(n=8)$ & $188 \pm 28^{*}$ & $261 \pm 34^{*}$ \\
KA + CORT + mannose $(n=7)$ & $93 \pm 28$ & $152 \pm 37$
\end{tabular}

$\overline{\text { Adrenalectomy and administration of KA, CORT, and sugars were as described }}$ in Materials and Methods. All sugars were administered in doses of $0.5 \mathrm{gm} / 3 \mathrm{hr}$, i.p., as described. Asterisk, significant difference from KA-treated group at 0.05 level (Scheffe post hoc test following 1-way ANOVA).

of brain fuels. Coadministration of glucose during the CORT treatment period partially protected the hippocampus. Equivalent doses of mannose were extremely effective in preventing the synergy. Double the dose of fructose, the only sugar of the 3 utilized that does not readily penetrate the blood-brain barrier, was required to reduce damage.

Continuous intracerebroventricular administration of the ketone $\mathrm{B}-\mathrm{OH}$ during the CORT treatment period was also effective in reducing the synergy (Fig. $2 B$ ). Representative photomicrographs are shown comparing damage induced by $3 A P$ plus CORT with or without B-OH (Fig. 1).

Kainic acid alone produced moderate damage in the hippocampus, centered in the CA3 pyramidal cell region (Table 2). This toxicity was exacerbated by CORT coadministration; as previously shown, the synergy was not as dramatic as between CORT and 3AP. [As evidence, in the case of KA, a significant difference is observed only when comparing adrenalectomized and CORT-treated rats, while with $3 \mathrm{AP}$, significant differences are found between adrenalectomized and intact, and between intact and CORT-trcatcd animals (Sapolsky, 1985a, b, in press). Thus, for convenience, intact and CORT-treated rats were compared in the 3AP experiment, while CORT-treated animals were compared with adrenalectomized rats in the KA experiment.] The synergy between CORT and KA could be attenuated by coadministration of mannose, while glucose administration was ineffective.

\section{Discussion}

The importance of the hippocampus in cognition, affect, and neuroendocrine regulation (Sapolsky et al., 1984; Scolville and Milner, 1957) underlines the importance of understanding the actions of GCs in damaging the hippocampus during normal aging and following neuropathologic insults. The present and previous reports suggest that GCs damage hippocampal neurons by impairing their capacities to survive a variety of insults. The differing mechanisms of action of these insults suggest that the synergy occurs because GCs act broadly to decrease neuronal viability, rather than because the intrinsic toxicity of the insults is increased by GCs. This is further supported by the failure of GCs to increase the binding or diffusion of KA (Sapolsky, 1985a). The GC effect on these neurons appears to be direct, rather than secondary to metabolic effects elsewhere in the body, as the synergy occurs in vitro with cultures of dispersed fetal hippocampal neurons (Sapolsky and Vale, unpublished observations). This GC action appears to be receptor-mediated, as the synergy is prevented by a GC-receptor antagonist (Sapolsky and Vale, unpublished observations). Despite the fact that hypoxia-ischemia, KA, and 3AP are maximally damaging in different hippocampal regions-CA1, $\mathrm{CA} 3$, and dentate gyrus, respective- 
ly-all 3 regions contain GC receptor concentrations far higher than outside the hippocampus (Sapolsky et al., 1983b).

In seeking to understand the mechanisms by which GCs endanger hippocampal neurons, the rapidity of the effect was informative. This and prior studies have demonstrated that as little as $24 \mathrm{hr}$ of exposure to elevated CORT titers bracketing 3AP or KA infusion can potentiate damage (Sapolsky, 1985b, in press). In the case of hypoxia-ischemia, the minimal period tested, $72 \mathrm{hr}$ of CORT administration following reperfusion, was sufficient to exacerbate damage. Such a rapid time course rules out a number of potential mechanisms of GC action, including the slow nonenzymatic addition of GCs to proteins that has been shown to occur and to potentially disrupt cell activity (Bucala et al., 1982). Instead, the present report suggests that the damaging aspects of GC exposure arise, at least in part, from more rapid effects of CORT on neuronal energy metabolism. As discussed in the introduction, neurons are extremely vulnerable to depletion of energy substrates, and all 3 insults are likely to produce such depletions. It appeared likely that GCs potentiate the damage of these insults by exacerbating the state of energy depletion in these neurons. GCs inhibit glucose uptake in peripheral tissues such as adipose, skin, and thymocytes (Munck, 1971). While measures of whole-brain glucose content have not indicated a similar GC effect throughout the entire organ (Watanabe and Passonneau, 1973), the steroids significantly inhibit glucose uptake and utilization in the hippocampus, as determined by both measurement of labeled glucose in dissected individual brain regions (Landgraf et al., 1978) and by the 2-deoxyglucose method (M. Kadekaro, personal communication). The vulnerabilities of cerebral metabolism, plus the actions of GCs and of the varied insults, suggested that supplementing rats with additional brain fuels might counteract the insult-GC synergy.

Glucose and mannose, both sugars that penetrate the bloodbrain barrier readily, were effective in reducing such synergy. Similarly, B-OH, a ketone body typically utilized by brain during starvation or diabetic ketoacidosis, attenuated synergistic damage. In contrast, higher concentrations of fructose, which does not readily penetrate the blood-brain barrier, were required to attenuate damage even minimally.

No difference in the severity of damage in rats given KA and those given KA plus mannose was observed (Table 2). This suggests that the brain fuels minimized the metabolic impact of the GCs, rather than the toxins. This must be a guarded interpretation: Since the damage induced by $3 \mathrm{AP}$ alone was too little to be attenuated by the addition of fuels, it is impossible to be certain if fuels reduce the toxicity of $3 \mathrm{AP}$ as well as the synergy between $3 \mathrm{AP}$ and GCs.

Glucose enters target cells via a saturable carrier-mediated system, and the GC inhibition of such transport appears to be noncompetitive (Munck, 1971). (It should be noted that these conclusions are based on study of thymus cells.) As such, the elevated glucose levels in glucose-treated rats were likely to partially, but not completely, override the actions of GCs in inhibiting uptake. Mannose, normally found in negligible concentrations in the bloodstream, can penetrate the blood-brain barrier, as can B-OH. They probably enter neurons via carriers, but there is no evidence that GCs inhibit their neuronal uptake (Siesjo, 1978). The differing efficacies of glucose and these 2 fuels in blocking the synergy (Fig. 2) may reflect the only partial success of the elevated glucose concentrations in overcoming the effects of $\mathrm{GC}$.

This discussion tacitly assumes that mannose is protective via its direct actions in the brain. Alternatively, one might speculate that mannose is utilized peripherally, freeing glucose for cerebral utilization. This is unlikely for 3 reasons. First, fructose would also substitute peripherally for glucose, yet it was less effective in attenuating hippocampal damage. Furthermore, administration of mannose (plus CORT) did not raise circulating glucose concentrations above those in rats administered CORT alone (Table 1). Finally, the protection of hippocampal neurons from the CORT-toxin synergy by mannose can be demonstrated with in vitro cultures of dispersed fetal hippocampal neurons (Sapolsky and Vale, unpublished observations). Thus, the protective actions of these brain fuels (and the implied endangering actions of GCs) appear to involve direct effects on hippocampal neuronal energy metabolism.

The present findings superficially appear to contradict those of Pulsinelli et al. (1982), who demonstrated that elevated circulating glucose concentrations are associated with enhanced hypoxic-ischemic injury. However, this glucose effect appears to be specific to that particular insult. During hypoxia-ischemia, cerebral metabolism is predominantly anaerobic, and the resultant accumulation of lactic acid appears to be one proximal agent of cell damage in this insult. Elevated glucose concentrations prior to hypoxia are thought to lead to increases in neuronal glycogen stores, which, in turn, are metabolized to damaging lactic acid during the hypoxia (cf. Siesjo, 1978). Thus, glucose concentrations following reperfusion do not influence neuropathologic outcome (Pulsinelli et al., 1982). There is no evidence that KA or $3 \mathrm{AP}$ damages the brain via lactic acidosis: the discrepancy with the ischemia literature is thus not surprising and suggests that the present findings are unlikely to extend to that model of hippocampal damage.

In a thoughtful review, Munck (1971) viewed the metabolic actions of GCs as part of a strategy of energy redistribution during stress. Energy utilization and storage are inhibited in fat, skin, thymocytes, and some brain regions by the breakdown of proteins and triglycerides (causing an efflux of amino acids and free fatty acids) and the blockade of glucose uptake. In concert with the direct and indirect GC stimulation of hepatic gluconeogenesis, these effects produce elevated circulating concentrations of glucose to be utilized by muscle-the tissue most likely to have an increased demand for energy during a variety of stressors, and whose glucose uptake is unaffected by GCs (Munck, 1971). It is therefore puzzling that neurological insults such as cerebral ischemia or seizure can provoke GC secretion as robustly as do stressors such as forced exercise or immobilization (Feibel et al., 1977). The responsiveness of the adrenocortical axis to purely neurological insults appears somewhat maladaptive, as energy utilization is dampened in critically vulncrable ncurons and favored instead in arguably irrelevant muscle. The well-established vulnerability of the hippocampus to these neuropathologic insults may thus be related, in part, to the catabolic actions of GCs and the extremely high concentrations of $\mathrm{GC}$ receptors in that brain region.

\section{References}

Aus der Muhlen, K., and H. Ockenfels (1969) Morphologische veranderungen im diencephalon und telencephalon nach storungen des regelkreises adenohypophyse-nebennierenrinde. III. Ergebnisse beim meerschweinchen nach verabreichung von cortison und hydrocortison. Z. Zellforsch. 93: 126-141.

Bucala, R., J. Fishman, and A. Cerami (1982) Formation of covalent adducts between cortisol and 16alpha-hydroxyestrone and protein: Possible role in the pathogenesis of cortisol toxicity and systemic lupus erythematosus. Proc. Natl. Acad. Sci. USA 79: 3320-3324.

Coyle, J. (1983) Neurotoxic action of kainic acid. J. Neurochem. 41 : $1-11$.

Davis, J., D. Wirtshafter, K. Asin, and D. Brief (1981) Sustained intracerebroventricular infusion of brain fuels reduces body weight and food intake in rats. Science 212: 81-83.

De Kosky, S., S. Scheff, and C. Cotman (1984) Elevated corticosterone levels: A possible cause of reduced axon sprouting in aged animals. Neuroendocrinology 38: 33-38.

Feibel, J., P. Hardy, R. Campbell, M. Goldstein, and R. Joynt (1977) Prognostic value of the stress response following stroke. JAMA 238: 1374-1376. 
Hicks, S. (1955) Pathologic effects of antimetabolites. 1. Acute lesions in hypothalamus, peripheral ganglia and adrenal medulla caused by 3-acetylpyridine and prevented by nicotinamide. Am. J. Pathol. 31: 189-197.

Krieger, D. (1982) Cushing's Syndrome (Monogr. Endocrinol., Vol. 22), p. 142, Springer-Verlag, Berlin.

Landfield, P., J. Waymire, and G. Lynch (1978) Hippocampal aging and adrenocorticoids: Quantitative correlations. Science 202: 10981102.

Landfield, P., R. Baskin, and T. Pitler (1981) Brain aging correlates: Retardation by hormonal-pharmacological treatments. Science 214: 581-584.

Landgraf, R., A. Mitro, and J. Hess (1978) Regional net uptake of ${ }^{14} \mathrm{C}$-glucose by rat brain under the influence of corticosterone. Endocrinol. Exp. 12: 119-128.

Lorente de No, R. (1934) Studies on the structure of the cerebral cortex. II. Continuation of the study of the ammonic system. J. Psychol. Neurol. 46: 113-177.

McEwen, B., J. Weiss, and L. Schwartz (1968) Selective retention of corticosterone by limbic structures in rat brain. Nature 220: 911912.

Munck, A. (1971) Glucocorticoid inhibition of glucose uptake by peripheral tissues: Old and new evidence, molecular mechanisms and physiological significance. Perspect. Biol. Med. 14: 265-289.

Munck, A., P. Guyre, and N. Holbrook (1984) Physiological functions of glucocorticoids in stress and their relation to pharmacological actions. Endocrinol. Rev. 5: 25-44.

Pulsinelli, W., S. Waldman, D. Rawlinson, and F. Plum (1982) Moderate hyperglycemia augments ischemic brain damage: A neuropathologic study in the rat. Neurology 32: 1239-1246.

Sapolsky, R. (1985a) A mechanism for glucocorticoid toxicity in the hippocampus: Increased neuronal vulnerability to metabolic insults. J. Neurosci. 5: 1227-1231.
Sapolsky, R. (1985b) Glucocorticoid toxicity in the hippocampus: Temporal aspects of neuronal vulnerability. Brain Res. 359: 300306.

Sapolsky, R. (in press) Glucocorticoid toxicity in the hippocampus: Synergy with an excitotoxin. Neuroendocrinology.

Sapolsky, R., and W. Pulsinelli (1985) Glucocorticoids potentiate ischemic injury to neurons: Therapeutic implications. Science 229: 13971400.

Sapolsky, R., L. Krey, and B. McEwen (1983a) The adrenocortical stress-response in the aged male rat: Impairment of recovery from stress. Exp. Gerontol. 18: 55-64.

Sapolsky, R., B. McEwen, and T. Rainbow (1983b) Quantitative autoradiography of ${ }^{3} \mathrm{H}$ corticosterone receptors in rat brain. Brain Res. 271: 331-337.

Sapolsky, R., L. Krey, and B. McEwen (1984) Glucocorticoid-sensitive hippocampal neurons are involved in terminating the adrenocortical stress-response. Proc. Natl. Acad. Sci. USA 81: 6174-6177.

Sapolsky, R., L. Krey, and B. McEwen (1985) Prolonged glucocorticoid exposure reduces hippocampal neuron number: Implications for aging. J. Neurosci. 5: 1221-1226.

Scolville, W., and B. Milner (1957) Loss of recent memory after bilateral hippocampal lesions. J. Neurol. Psychiatr. 20: 11-21.

Siesjo, B. (1978) Brain Energy Metabolism, Wiley, New York.

Siesjo, B. (1981) Cell damage in the brain: A speculative synthesis. J. Cerebral Blood Flow Metabol. I: 155-185.

Tang, G., and R. Phillips (1978) Some age-related changes in pituitaryadrenal function in the male laboratory rat. J. Gerontol. 33: 377-382.

Theoret, Y., J. Caldwell-Kenkel, and M. Krigman (1985) The toxicologist (in press)

Watanahe, H., and J. Passonneau (1973) Factors affecting the turnover of cerebral glycogen and limit dextrin in vivo. J. Neurochem. 20:15431554. 